\title{
Fetal parathyroids are not required to maintain placental calcium transport
}

\author{
Christopher S. Kovacs, ${ }^{1}$ Nancy R. Manley, ${ }^{2}$ Jane M. Moseley, ${ }^{3}$ T. John Martin, ${ }^{3}$ \\ and Henry M. Kronenberg 4
}

${ }^{1}$ Faculty of Medicine-Endocrinology, Memorial University of Newfoundland, St. John's, Newfoundland, Canada ${ }^{2}$ Institute of Molecular Medicine and Genetics, Department of Pediatrics, Medical College of Georgia, Augusta, Georgia, USA ${ }^{3}$ St. Vincent's Institute for Medical Research, University of Melbourne, Fitzroy, Victoria, Australia

${ }^{4}$ Endocrine Unit, Massachusetts General Hospital and Harvard Medical School, Boston, Massachusetts, USA

Address correspondence to: Christopher S. Kovacs, Faculty of Medicine-Endocrinology, Memorial University of Newfoundland, 300 Prince Philip Drive, St. John's, Newfoundland A1B 3V6, Canada. Phone: (709) 777-6881; Fax: (709) 777-8049; E-mail ckovacs@mun.ca.

Received for publication September 13, 2000, and accepted in revised form March 13, 2001.

We used Hoxa3 knockout mice and other mouse models to study the role of the fetal parathyroids in fetal calcium homeostasis. Hoxa3-null fetuses lack parathyroid glands, and absence of parathyroid hormone $(\mathrm{PTH})$ was confirmed with a rodent $\mathrm{PTH}$ immunoradiometric assay. The ionized calcium level of Hoxa3-null fetuses was significantly lower than that of wild-type or heterozygous littermates or of the mother. Both the rate of placental calcium transfer and the plasma PTHrP level were normal in Hoxa3 mutants and their heterozygous siblings. Because we had previously observed an increase in placental calcium transfer in $P T H / P T H r P$ receptor 1 -null (Pthr1-null) fetuses, we assayed plasma PTHrP in those mice. Pthr1-null fetuses had plasma PTHrP levels 11-fold higher than those of their littermates. Northern analysis, immunohistochemical, and in situ hybridization studies of Pthr1-null fetuses indicated that liver and placenta had increased expression of PTHrP. In summary, loss of fetal parathyroids in Hoxa3-null fetuses caused marked hypocalcemia but did not alter placental calcium transfer or the circulating PTHrP level. The findings in the Pthr1-null fetuses indicate that several tissues may contribute to the circulating PTHrP level in fetal mice.

J. Clin. Invest: 107:1007-1015 (2001).

\section{Introduction}

Fetal calcium metabolism differs from that of adults in several ways, reflecting the unique needs of the developing fetus (1). Calcium is actively transported across the placenta, and the fetus normally has a higher blood calcium than does the mother, resulting in a maternalfetal calcium gradient. In the adult, parathyroid hormone $(\mathrm{PTH})$, acting through the $\mathrm{PTH} / \mathrm{PTHrP}$ receptor, is the major regulator of blood calcium. In the fetus, however, PTH and parathyroid hormone-related protein (PTHrP) share in the regulation of the fetus's unique calcium metabolism, acting through the $\mathrm{PTH} / \mathrm{PTHrP}$ receptor and other receptors. PTHrP may play a dominant role in regulating fetal-placental calcium homeostasis, while PTH is normally suppressed in late gestation and its importance to fetal calcium homeostasis has been less clearly defined.

We have previously shown that absence of PTHrP (in the Pthrp-null fetus; ref. 2) reduced the blood calcium to the maternal level (zero maternal-fetal gradient), and reduced the placental transfer of ${ }^{45} \mathrm{Ca}(3)$. Further, we found that administration of PTHrP 1-86 or PTHrP 6786 (but not PTH 1-84 or PTH 1-34) increased the rate of placental calcium transfer in Pthrp-null fetuses. These experiments were consistent with the previous finding that mid-molecular fragments of PTHrP (but not intact PTH) could stimulate placental calcium transfer in fetal lambs that had been thyroparathyroidectomized $(4,5)$. In contrast to the findings in Pthrp-null fetuses, we had noted in $P T H / P T H r P$ receptor 1-null (Pthr1-null) fetuses (6) that the fetal blood calcium was reduced below that of the mother (reversed maternal-fetal gradient) but that placental calcium transfer was increased to about $150 \%$ of the value of the heterozygote littermates (3).

These experiments confirmed that PTHrP plays an important role in normal fetal-placental calcium homeostasis, given that in the absence of PTHrP, the fetus is hypocalcemic, hyperphosphatemic, and has reduced placental calcium transfer. Pthrp-null fetuses have markedly elevated serum PTH levels (7); evidently, however, PTH is unable to compensate completely for lack of PTHrP. In fact, when PTH is further stimulated in the Pthrp-null fetuses by simultaneously deleting the Calcium sensing receptor (Casr) gene (8), the blood calcium of such $\mathrm{Pthrp}^{-/-} \mathrm{Casr}^{-/}$fetuses is higher than that of the pure Pthrp-null fetus, but the rate of placental calcium transfer is still reduced in $\mathrm{Pthrp}^{-/-} \mathrm{Casr}^{--}$ fetuses (9).

The observations in the Ptbr1-null fetuses suggested that PTH does have a role in maintaining the fetal blood calcium, as the blood calcium in these fetuses was lower than that in Pthrp-null fetuses. The increased placental calcium transfer in Pthr1-null fetuses was 
interpreted to indicate possible upregulation of PTHrP, and, in particular, the PTHrP mid-molecule that regulates placental calcium transfer (3).

We address the remaining important questions about the relative roles of PTHrP and PTH in regulating fetal calcium homeostasis: First, whether PTH plays an important role in fetal calcium homeostasis, and second, what tissue source(s) of PTHrP is important for fetal calcium homeostasis. Regarding the latter question, indirect evidence suggests that the parathyroids are a major source of fetal blood PTHrP in sheep $(4,5)$, but direct confirmation of this hypothesis is lacking. In fact, a detailed study of the parathyroids of fetal rats found abundant PTH mRNA but no detectable PTHrP mRNA (10), suggesting that the fetal parathyroids are not the source of PTHrP in the circulation of that species. PTHrP is expressed widely in fetal tissues (including the placenta) $(1,11)$, any number of which might contribute to the circulating PTHrP level and the regulation of placental calcium transfer.

To address both questions, we utilized several mouse models, including a model in which parathyroid glands are missing as a consequence of a genetic deletion (12). In the latter model, Hoxa3-null fetuses die soon after birth, with abnormalities in tissues derived from the third and fourth pharyngeal arches, including thyroid hypoplasia, deletion of the thymus, and (most relevant to our studies) absence of parathyroids. Thus, in addition to lacking PTH, these null mice should lack PTHrP that is derived from the parathyroids, but should retain PTHrP that is derived from the placenta and other fetal tissue sources. This model enabled us to examine the physiological contribution of the parathyroids to fetal calcium metabolism, and, in particular, to determine whether that role is accomplished through PTH, PTHrP, or both. By comparing and contrasting the effects of loss of parathyroids (in Hoxa3-null) to loss of PTHrP (in Pthrp-null) and resistance to PTH action (in Pthr1-null), both the role of PTH and the source(s) of PTHrP relevant to fetal calcium metabolism were examined.

We find that lack of parathyroids in Hoxa3-null fetuses leads to absence of circulating PTH and clear disruptions in mineral metabolism that establish a definite role for PTH in normal fetal calcium homeostasis. However, absence of parathyroids did not alter serum PTHrP levels or the rate of placental calcium transfer, indicating that the parathyroids may not contribute significantly to the circulating PTHrP level or the regulation of placental calcium transfer. We then re-examined the Pthr 1 model (in which placental calcium transfer is upregulated) and found increased serum PTHrP levels and evidence of upregulation of PTHrP by fetal liver and placenta. Altogether, the observations in these contrasted mouse models indicate that the parathyroids do play a role in fetal calcium metabolism through the production of PTH but possibly not PTHrP, whereas placenta and liver (and possibly other fetal tissues) may contribute to fetal calcium metabolism through the production of PTHrP.

\section{Methods}

Knockout mice and genotyping. Hoxa3, Pthrp, Pthr1, and Casr gene knockout mice were obtained by targeted disruption of the murine genes in embryonic stem cells, as described previously $(2,6,8,12,13)$. Within-knockout comparisons were made among fetal littermates only. Each strain was back-crossed into Black Swiss (Taconic Inc., Germantown, New York, USA) for at least three generations to provide a comparable genetic background among the colonies. Mice were mated overnight; the presence of a vaginal mucus plug on the morning after mating marked gestational day 0.5 . Normal gestation in these mice is 19 days. All mice were given a standard chow diet and water. All studies were performed with the prior approval of the Institutional Animal Care and Use Committee of the Massachusetts General Hospital, and the Institutional Animal Care Committee of Memorial University of Newfoundland.

Genomic DNA was obtained from fetal tails, and genotyping was accomplished by PCR using primers that were specific to the Hoxa3, Pthrp, Pthr1, and Casr gene sequences $(3,9,12)$, in a single-tube, 36-cycle PCR reaction utilizing a PTC-200 Peltier Thermal Cycler (MJ Research, Cambridge, Massachusetts, USA). For the double-knockout mice, genotyping of the Casr alleles and Hoxa3 alleles was accomplished in two separate PCR reactions.

Whole blood and amniotic fluid collection. In general, fetal blood was drawn on day 18.5 of gestation to maximize the volume of serum or whole blood obtained. Pregnant mice were sacrificed by cervical dislocation to avoid the effects of anesthesia and hypoventilation on the ionized calcium, pH, and PTH. The neck of each individual fetus was incised. Whole blood ( 40-75 $\mu \mathrm{l}$ obtainable per fetus) was collected into heparinized capillary tubes for ionized calcium measurements and into plain capillary tubes (no anticoagulant) for serum collection. For plasma PTHrP measurements, whole blood was collected into plain capillary tubes that had been flushed first with an EDTA-aprotinin buffer $(0.5$ $\mathrm{M} \mathrm{Na}{ }_{2}$ EDTA and 40 TI units per milliliter of aprotinin). Heparinized samples were kept on ice until used. All other samples were separated by centrifugation; sera or plasma was then stored at $-20^{\circ} \mathrm{C}$ until assayed.

Amniotic fluid was collected from fetuses on day 17.5 of gestation, as the amniotic fluid was generally too scanty and viscous on day 18.5. Individual gestational sacs were lanced, and amniotic fluid was collected into heparinized $100 \mu \mathrm{l}$ capillary tubes and stored at $-20^{\circ} \mathrm{C}$ until used.

Maternal blood was collected into capillary tubes from the tail vein, immediately before a caesarian section on day $18 \pm 0.5$ of pregnancy.

Placental calcium transport. This procedure has been described in detail elsewhere (3). Briefly, pregnant dams on day $17 \pm 0.5$ of gestation were given an intracardiac injection of $50 \mu \mathrm{Ci}{ }^{45} \mathrm{Ca}$ and $50 \mu \mathrm{Ci}{ }^{51} \mathrm{Cr}$-EDTA. Five minutes later, the dam was sacrificed, and each fetus was removed. The ratio of ${ }^{45} \mathrm{Ca} /{ }^{51} \mathrm{Cr}$ radioactivity was determined for each fetus using a $\gamma$-counter and a liquid scin- 


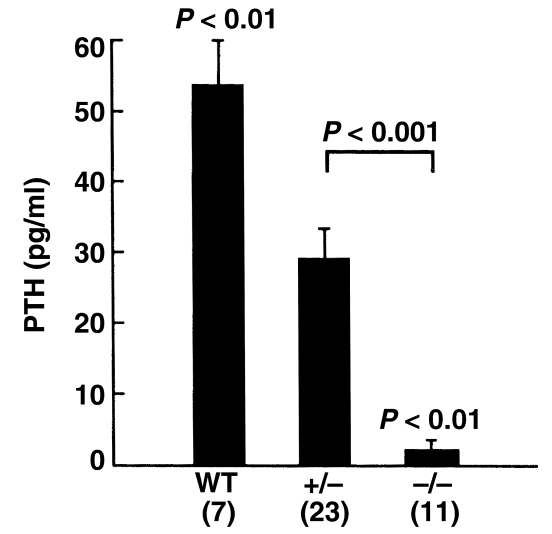

Figure 1

Serum PTH. Serum PTH level in Hoxa3-null fetuses and littermates (embryonic day [ED] 18.5). Heterozygous and null values were each significantly lower than wild-type. The number of observations for each genotype is indicated in parentheses.

tillation counter, respectively. The data were normalized to the mean ${ }^{45} \mathrm{Ca} /{ }^{51} \mathrm{Cr}$ activity ratio of the $\mathrm{Hoxa}^{+/}{ }^{+/}$fetuses in each litter, so that the results from different litters could be compared. Owing to the effect of rounding of the individual normalized values, the overall mean for all $\mathrm{Hoxa}^{+}{ }^{+-}$- fetuses was not exactly $100 \%$.

Chemical assays. Ionized calcium was measured on whole blood using a Chiron Diagnostics $634 \mathrm{Ca}^{++} / \mathrm{pH}$ Analyzer (Chiron Diagnostics, East Walpole, Massachusetts, USA). Total calcium and magnesium were determined on serum and/or amniotic fluid using photometric assays (Sigma-Aldrich, Oakville, Ontario, Canada). Serum PTH was measured using a rodent PTH 1-34 immunoradiometric assay (IRMA) kit (Nichols Institute, San Juan Capistrano, California, USA); the stated detection limit of the assay was 2.0 $\mathrm{pg} / \mathrm{ml}$. Calcitonin was measured with an IRMA specific for rat calcitonin (Immutopics, San Clemente, California, USA) (14). In lieu of mouse calcitonin standards, values were expressed as pg-equivalents rat calcitonin $/ \mathrm{ml}$; the stated detection limit of the assay was $1.0 \mathrm{pg} / \mathrm{ml}$. Plasma PTHrP was measured using a sensitive RIA with an $\mathrm{NH}_{2}$-terminal epitope; the detection limit of this assay in human plasma is nor- mally 2 pmol/l (15). When applied to the assay of mouse plasma, assay buffer was used instead of human plasma as diluents for standards. Using this assay, in Pthrp-null fetuses a reading of $3.8 \pm 0.3$ $\mathrm{pmol} / \mathrm{l}$ was obtained for plasma, significantly lower $(P$ $<0.001$ ) than the reading of $5.7 \pm 0.2 \mathrm{pmol} / \mathrm{l}$ in the littermates. Assuming no circulating PTHrP in the Pthrp-null fetuses, and given that the standard curve contains no plasma, the level of $3.8 \mathrm{pmol} / \mathrm{l}$ reflects nonspecific inhibition of antibody binding by plasma and may approximate the detection limit of the assay under these conditions. For the PTH, PTHrP, and calcitonin assays, three or four samples of mouse serum were pooled together to obtain sample volumes of 100 $\mu l$. These pooled samples were then diluted (if necessary) with zero standard to meet the sample size requirements of the assay.

Tissue collection. For total RNA, the following fetal tissues were taken from embryonic day (ED) 18.5 fetuses: head, neck (to represent the parathyroids), lung, liver, umbilical cord, and placenta. The tissues were harvested quickly after a rapid cervical dislocation and caesarean section, and the tissues were snap-frozen in liquid nitrogen before being placed at $-80^{\circ} \mathrm{C}$ for storage. For immunohistochemistry and in situ hybridization, fetal liver, placenta, and maternal liver (control) were harvested. The liver was placed directly into formalin for initial fixation, whereas the placentas were obtained after placental perfusion with paraformaldehyde (see later here).

Placental fixation by perfusion. Fixation by placental perfusion with paraformaldehyde was carried out to minimize the degradation in mRNA or protein levels that might occur during fixation and processing of the RNase and protease-rich placental tissues (16). Subsequently, the placentas were removed and placed in 10\% formalin for standard processing, embedding in paraffin, and sectioning.

Riboprobe and DNA probe labeling. For in situ hybridization, the plasmids were linearized with appropriate restriction enzymes, and labeled with $125 \mu \mathrm{Ci}$ of ${ }^{35} \mathrm{~S}$ UTP using an SP6/T7 Transcription Kit (Promega/Fisher Scientific Ltd., Burlington, Ontario, Canada), and the appropriate polymerase. Unincorporated nucleotides were removed with the NucTrap columns (Stratagene, La Jolla, California, USA) per package instructions.
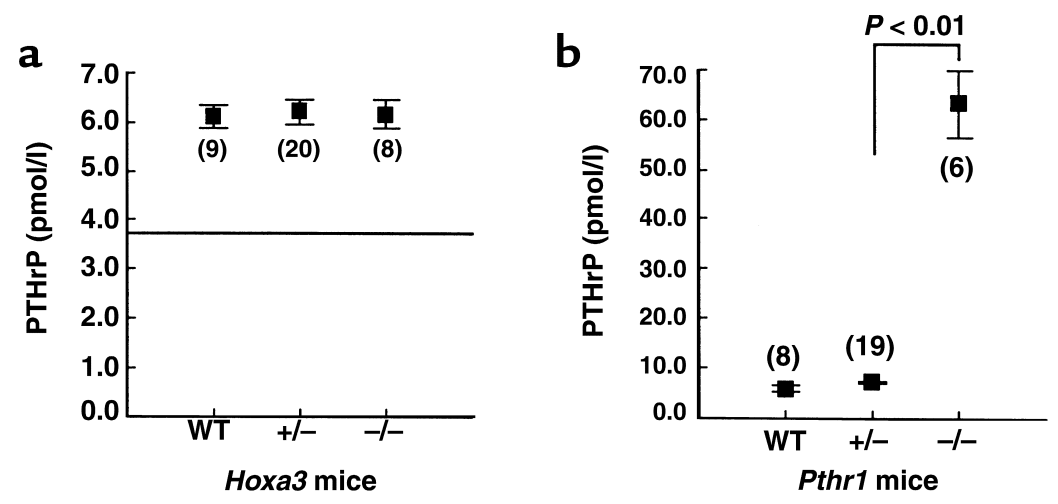
Figure 2
Plasma PTHrP. Plasma PTHrP 1-34 level in (a) Hoxa3-null fetuses and (b) Pthr1-null fetuses and their corresponding littermates (ED 18.5). The number of observations for each genotype is indicated in parentheses. The horizontal line in $\mathbf{a}$ indicates the plasma PTHrP 1-34 level of Pthrp-null fetuses.


For Northern blots, the insert was cut out of the plasmid and purified using an Ultrafree-MC spin column (Millipore Corp., Bedford, Massachusetts, USA). A standard random priming protocol, with the DNA polymerase Klenow, was used to generate $\alpha-{ }^{32} \mathrm{P}$-labeled DNA probes. Unincorporated nucleotides were removed with NucTrap columns.

A plasmid containing a cDNA for full-length rat PTHrP was used for in situ hybridization and the Northern blot. A plasmid containing a human cDNA for Cyclophilin (Cyclo) was used to verify total RNA loading on the Northern blot.

Northern blot analysis. Frozen tissue samples were placed directly into Trizol reagent, and homogenized for 1 minute with a tissue homogenizer at high speed. Total cellular RNA isolation was performed according to the protocol that accompanies the Trizol reagent. RNA samples were transferred to a MOPS-formaldehyde agarose gel, electrophoresed, and transferred from the gel to nitrocellulose blots. Blots were hybridized (50\% formamide, $4 \times$ SSC, $7 \mathrm{mM}$ Tris, $10 \%$ dextran sulfate, $100 \mu \mathrm{g} / \mathrm{ml}$ salmon sperm DNA, $2 \times$ Denhardt's) with $10^{6}-10^{7} \mathrm{cpm}$ of probe for $18-20$ hour at $70^{\circ} \mathrm{C}$ in a hybridization oven, washed $(2 \times$ SSC in $0.1 \%$ SDS; $1 \times$ SSC in $0.1 \%$ SDS; $0.5 \times \mathrm{SSC}$ in $0.1 \% \mathrm{SDS}$; at $55^{\circ} \mathrm{C}$ for 30 minutes each), and exposed to $\mathrm{x}$-ray film at $-80^{\circ} \mathrm{C}$. Four separate litters of fetal mice were used independently in these experiments.

In situ bybridization. In situ hybridization was performed on $5-\mu \mathrm{m}$ tissue sections as described previously (17). Hybridization was performed in a humidified chamber (for 16 hours at $55^{\circ} \mathrm{C}$ ) with the labeled riboprobe diluted 1:20 in the hybridization solution. Sections were successively washed, RNase treated, and dehydrated in graded ethanol series. An overnight exposure of the slides to plain x-ray film enabled an estimate of exposure time for the liquid emulsion step. Slides were then dipped into NTB-2 liquid emulsion, dried, stored in light-tight boxes, and kept at $4^{\circ} \mathrm{C}$ until developed (2-6 weeks). The emulsion was developed using standard developer and fixer, and the sections were then counterstained with hematoxylin-eosin.
All comparisons of wild-type to null were made between tissues obtained from within the same litter and that had been processed, embedded, and sectioned at the same time. Also, such comparative sections were always hybridized together with the same probe and washed together, to validate the comparison and to minimize artifacts. Assessments of signal intensity were determined in a blinded fashion (no knowledge of the genotype). The reproducibility of the results was confirmed independently on at least three separate litters.

Immunohistochemistry. PTHrP immunohistochemistry was performed using standard immunohistochemical technique with the following specific adaptations: Washes consisted of PBS containing 5\% newborn bovine serum (PBS/NBS) (Life Technologies Inc., Burlington, Ontario, Canada). Nonspecific staining was blocked with normal goat serum diluted in PBS/NBS. Primary antibody was rabbit anti-PTHrP[1-14] (18), diluted 1:200 in PBS/NBS, and applied at $20^{\circ} \mathrm{C}$ for 60 minutes (this step was omitted from control sections). Biotinylated secondary antibody was goat anti-rabbit (Vector Canada, Burlington, Ontario, Canada), diluted in PBS/NBS, and applied at $20^{\circ} \mathrm{C}$ for 30 minutes. $\mathrm{ABC}$ reagent (Vector) was diluted in PBS and applied at $20^{\circ} \mathrm{C}$ for $30 \mathrm{~min}-$ utes. DAB-Tris-peroxidase substrate (Vector) was placed over the sections, and the reaction was allowed to develop for 3-10 minutes. Sections were counterstained with Contrast Red (Life Technologies Inc.), washed, dehydrated, and mounted. Assessments of staining intensity were determined in a blinded fashion (no knowledge of the genotype). The reproducibility of the results was confirmed independently on at least three separate litters. Pthrp-null tissues of similar archival age were used as independent controls for antibody specificity; such tissues were treated and stained together with the wild-type and Pthr 1 tissues with which they were compared.

Statistical analysis. Data were analyzed using SYSTAT 5.2.1 for Macintosh (SYSTAT Inc., Evanston, Illinois, USA). ANOVA was used for the initial analysis; Tukey's test was used to determine which pairs of means differed significantly from each other. Two-tailed probabilities are reported, and all data are presented as mean \pm SE.

Table 1

Mineral and hormonal characteristics, and ${ }^{45} \mathrm{Ca} /{ }^{51} \mathrm{Cr}$ placental transfer, of Hoxa3-null fetuses compared to their littermates ${ }^{\mathrm{A}}$

\begin{tabular}{|c|c|c|c|c|}
\hline & WT & $+/-$ & $-1-$ & $\begin{array}{c}P \\
(-/- \text { versus }+/-)\end{array}$ \\
\hline Serum calcitonin $(\mathrm{pg} / \mathrm{ml})$ & $69.7 \pm 11.6$ & $92.4 \pm 8.6$ & $89.4 \pm 12.8$ & Not significant \\
\hline lonized calcium ( $\mathrm{mmol} / \mathrm{l})$ & $1.47 \pm 0.02$ & $1.48 \pm 0.02$ & $1.07 \pm 0.03$ & $<0.001$ \\
\hline Serum calcium (mmol/l) & $1.98 \pm 0.08$ & $1.83 \pm 0.09$ & $1.47 \pm 0.09$ & $<0.002$ \\
\hline Serum magnesium (mmol/l) & $1.22 \pm 0.07$ & $1.23 \pm 0.07$ & $1.00 \pm 0.07$ & $<0.05$ \\
\hline Serum phosphate $(\mathrm{mmol} / \mathrm{l})$ & $2.73 \pm 0.18$ & $2.88 \pm 0.18$ & $3.44 \pm 0.18$ & $<0.03$ \\
\hline Amniotic calcium ( $\mathrm{mmol} / \mathrm{l})$ & $1.70 \pm 0.10$ & $1.77 \pm 0.10$ & $1.32 \pm 0.11$ & $<0.008$ \\
\hline $\begin{array}{l}{ }^{45} \mathrm{Ca} /{ }^{51} \mathrm{Cr} \text { placental transfer } \\
\text { (expressed as percent } \\
\text { of heterozygous mean) }\end{array}$ & $101.2 \pm 7.9$ & $100.6 \pm 6.8$ & $104.5 \pm 10.8$ & Not significant \\
\hline
\end{tabular}

AAll measurements were obtained from ED 18.5 fetuses, except amniotic fluid and placental transfer measurements were done in ED 17.5 fetuses. Heterozygous values did not differ significantly from wild-type. $P$ values for heterozygous versus null are quoted; $P$ values of wild-type versus null were of similar magnitude. 
a

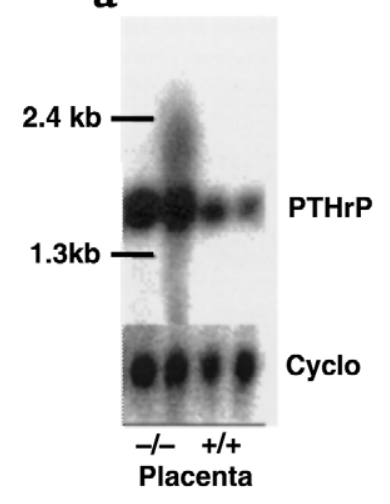

b

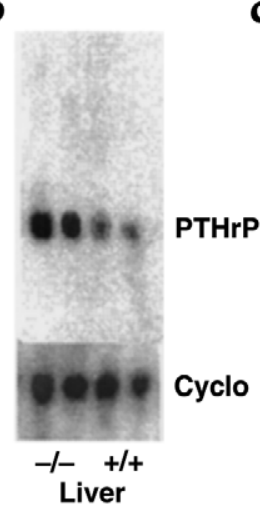

C

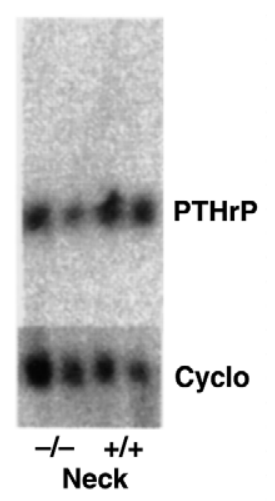

\section{Figure 3}

Northern analysis of PTHrP mRNA levels. Total RNA obtained from (a) placenta, (b) fetal liver, and (c) neck in Pthr1 and wild-type littermates (ED 18.5) and probed for PTHrP mRNA. Cyclophilin (Cyclo) was used as a loading control.

tion, Pthr1-null fetuses had an ionized calcium that was lower than the maternal ionized calcium concentration, such that maternal-fetal calcium gradient was reversed. Therefore, the ionized calcium level of Hoxa3-null fetuses is closer to that of Pthr1-null fetuses and suggests that the lower serum calcium cannot be explained by loss of PTHrP alone.

\section{Results}

$P T H$ and calcitonin secretion in Hoxa 3 mutant fetuses. A previous histological study of Hoxa3-null fetuses had failed to detect any recognizable parathyroid tissue and had suggested that the thyroid tissue (including $\mathrm{C}$ cells) was hypoplastic (12). To demonstrate that Hoxa3null fetuses lack all parathyroid cells capable of secreting PTH, the serum PTH level was assayed. As seen in Figure 1, the serum PTH level was undetectable in Hoxa3-null fetuses and was reduced in the heterozygotes compared with the wild-type. In contrast, assay of the serum calcitonin level showed no significant differences among the genotypes (Table 1). The normal calcitonin levels in the Hoxa3-null fetuses make it unlikely that hypoplasia of the thyroidal C cells would have a significant impact on calcium and bone metabolism in these fetuses. These data demonstrated that Hoxa3-null fetuses lack serum PTH and, therefore, probably all parathyroid cells.

That the Hoxa3-null mice lack PTH is a distinguishing characteristic from the recently described $\mathrm{Gcm} 2$ knockout mice, which also lack parathyroids but have normal circulating PTH levels due to production of PTH by the thymus (19). The absence of the parathyroids and thymus in the Hoxa3-null mice explains the complete lack of serum PTH in this model. It is also apparent from these results that loss of one Hoxa3 allele is sufficient to affect parathyroid hormone levels; this finding underscores the importance of Hoxa3 in fetal parathyroid formation and function.

Fetal mineral metabolism in Hoxa3-null fetuses. The aparathyroid Hoxa3-null fetuses had an ionized calcium of $1.07 \pm 0.03 \mathrm{mmol} / \mathrm{l}$, which was significantly lower than the ionized calcium of the littermates (Table 1). The maternal-fetal calcium gradient was obtained by subtracting the maternal ionized calcium level (1.24 $\pm 0.02 \mathrm{mmol} / \mathrm{l}$ ) from the fetal level. Although wild-type and heterozygous fetuses had an average calcium gradient of $+0.24 \mathrm{mmol} / \mathrm{l}$, the gradient was reversed to $-0.17 \mathrm{mmol} / \mathrm{l}$ in Hoxa3-null fetuses.

We previously reported that the ionized calcium of Pthrp-null fetuses was reduced to a level equal to the maternal ionized calcium, and that, as a consequence, the maternal-fetal calcium gradient was zero (3). In addi-
The biochemical phenotype of the Hoxa3-null fetuses was further characterized as shown in Table 1 . The total calcium level was also significantly lower in Hoxa3-null fetuses, whereas the serum phosphate level was increased. The serum magnesium was reduced in parallel with the serum calcium level. Amniotic fluid was collected as a surrogate for fetal urine, and the calcium content of amniotic fluid was found to be significantly reduced in the Hoxa3-null fetuses. The low amniotic fluid calcium content of the Hoxa3-null fetuses is consistent with the reduced serum ionized calcium and renal filtered load of calcium; in contrast, Pthrp-null fetuses (whose blood calcium is not as low as in Hoxa3-null) have unaltered amniotic calcium levels (data not shown). The raised serum phosphate is consistent with hypoparathyroidism and suggests that the fetal parathyroids may control the fetal serum phosphate (although Pthrp-null mice also have similarly raised serum phosphate levels).
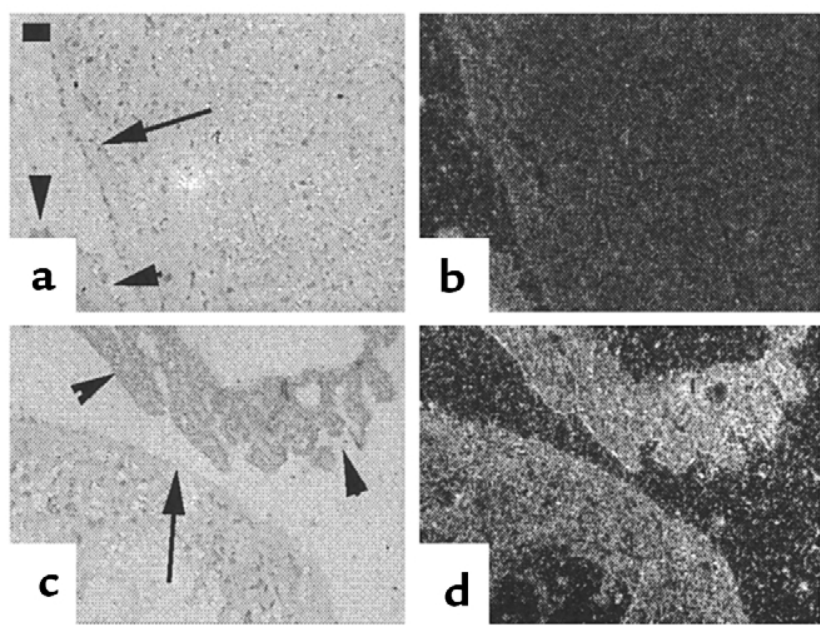

\section{Figure 4}

PTHrP in situ hybridization of placenta. Respective bright-field and dark-field images of placenta (ED 18.5) from wild-type (a and $\mathbf{b}$ ) and Pthr1-null mice (c and $\mathbf{d}$ ), probed with a ${ }^{35}$ S-labeled antisense probe, demonstrated diffusely increased expression of PTHrP mRNA in the Pthr1-null placenta and yolk sac. Arrows indicate the outer rim of the placenta; arrowheads indicate the visceral (extraplacental) yolk sac. Calibration bar $=50 \mu \mathrm{m}$. 

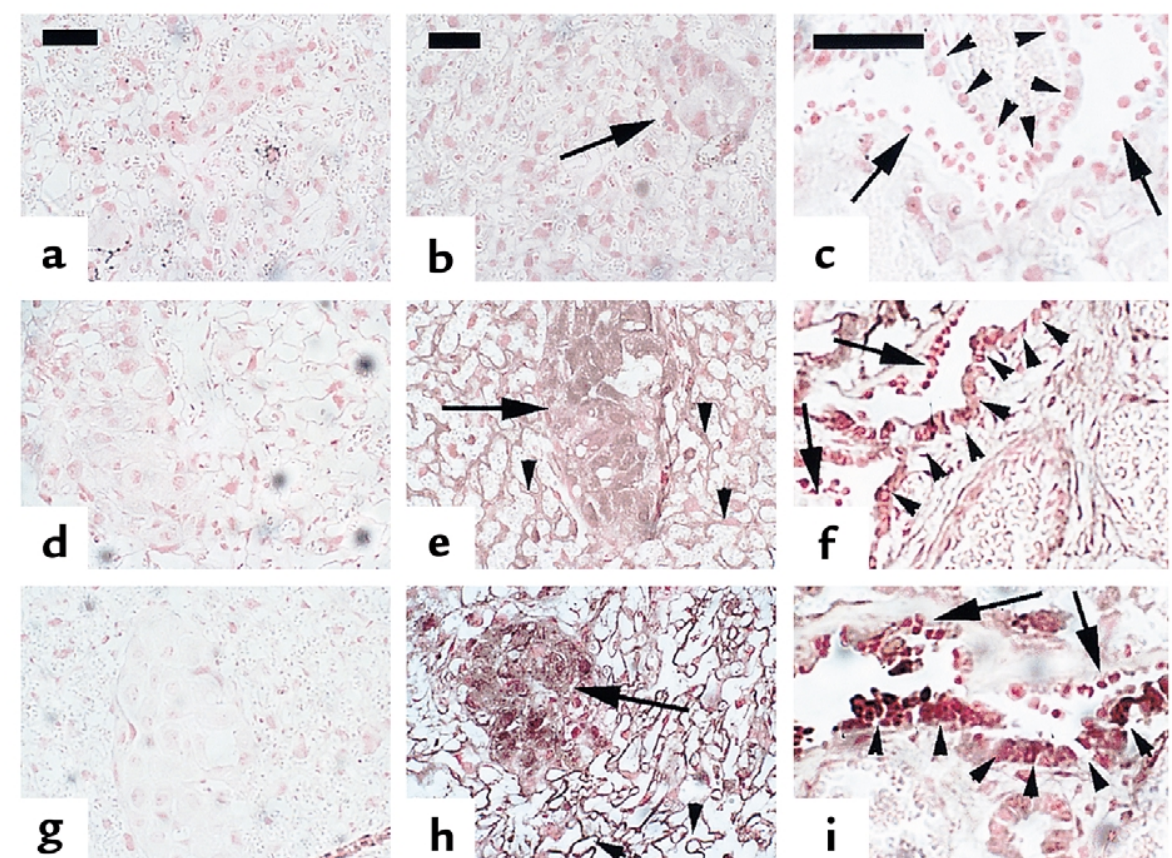

Figure 5

PTHrP immunohistochemistry of placenta. Upper images (a-c) are from Pthrp-null, middle images (d-f) are from wild-type, and lower images (g-i) are from Pthr1-null placentas (ED 18.5). a, d, and $\mathbf{g}$ are control sections in which the primary antibody to PTHrP 1-34 was omitted. Pthrp-null sections are controls to demonstrate the antibody specificity. In $\mathbf{h}$, PTHrP expression is higher in the labyrinthine (syncytial) trophoblasts (arrowheads) and spongiotrophoblasts (arrows) of Pthr1-null than in the wild-type (e); corresponding section of Pthrp-null (b) shows only nonspecific background. (i) Detail of the bilayered intraplacental yolk sac (IPYS) demonstrating PTHrP immunostaining in the columnar (visceral) cells of the IPYS (arrowheads), with less intense PTHrP immunostaining in the cuboidal (parietal) cells of the IPYS (arrows). Reichert's membrane underlies the cuboidal cells in all sections. A corresponding section of wild-type IPYS (f) shows less intense PTHrP immunostaining. Calibration bars $=50 \mu \mathrm{m}$.

Fetal mineral metabolism in Hoxa3-Casr double mutants. We previously reported that Casr-null fetuses are hypercalcemic in utero with marked hyperparathyroidism and elevated PTH levels (9). Further, double-knockout Pthrp ${ }^{-/-} \mathrm{Casr}^{-/-}$fetuses had a higher ionized calcium than the Pthrp-null alone (presumably due to hyperparathyroidism as a consequence of the disrupted Casr). To validate further the hypothesis that Hoxa3null fetuses lacked parathyroid cells and to demonstrate the role of PTH in mediating the phenotype of the Casr knockout mouse, double-knockout $\mathrm{Hoxa3}^{-/-} \mathrm{Casr}^{-1}$ fetuses were created. In this background, wild-type fetuses had an ionized calcium of $1.72 \pm 0.18 \mathrm{mmol} / \mathrm{l}$. By comparison, single mutant $\mathrm{Hoxa3}^{-/-}$fetuses had an ionized calcium of $1.41 \pm 0.08$

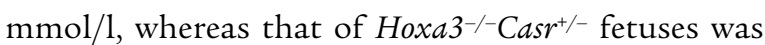
$1.43 \pm 0.09 \mathrm{mmol} / \mathrm{l}$, and of Hoxa $3^{-/-} \mathrm{Casr}^{-/}$double knockout fetuses was $1.40 \pm 0.10 \mathrm{mmol} / \mathrm{l}(P=$ not significant). The ionized calcium of Hoxa3-null fetuses was unaltered by a disrupted Casr, consistent with the absence of parathyroids, and thereby the inability to respond to a Casr mutation.

Placental calcium transfer in Hoxa3 mutant. The lower blood calcium of Hoxa3-null fetuses could result from such diverse mechanisms as reduced placental calcium transfer, decreased skeletal calcium resorption, increased mineral accretion by the skeleton, decreased renal tubular reabsorption of calcium, and absence of the parathyroid calcium receptor. We examined the contribution of placental calcium transfer by intracardiac injection of pregnant mice with ${ }^{45} \mathrm{Ca}$ and ${ }^{51} \mathrm{Cr}$-EDTA (3). Using this technique, the placental calcium transfer of Hoxa3-null fetuses was no different from that of the heterozygous and wt littermates (Table 1, bottom). Therefore, loss of fetal parathyroids did not affect placental calcium transfer in the Hoxa3-null fetuses.

Fetal plasma PTHrP in Hoxa3 and Pthr1 mutants. Because absence of the parathyroids in the Hoxa3-null fetuses did not impair placental calcium transfer, the parathyroids, and PTH in particular, are not required for maintenance of normal placental calcium transfer. If the parathyroids are an important source of PTHrP in the circulation, absence of the parathyroids might alter the circulating level of PTHrP. To assess this, we measured PTHrP 1-34 in fetal plasma on day 18.5 of gestation. Hoxa3-null fetuses had a plasma PTHrP level that was no different from that of the heterozygous and wild-type littermates (Figure 2a). Thus, loss of fetal parathyroids did not affect either the plasma PTHrP 134 level or the rate of placental calcium transfer.

To validate further the significance of the unaltered plasma PTHrP 1-34 level and the sensitivity of the assay under these experimental conditions, we next turned to the Pthr 1 knockout model. We had previously observed that Pthr1-null fetuses had a placental calcium transfer rate that was upregulated to about $150 \%$ of the het- 
erozygous and wild-type value (3). We had speculated that this might reflect upregulation of PTHrP and its effects on placental calcium transfer. Therefore, we next measured plasma PTHrP 1-34 in Pthr1-null fetuses and found the level to be $63.5 \pm 2.9 \mathrm{pmol} / \mathrm{l}$, a value 11 -fold that of heterozygous and wild-type littermates (Figure $2 b)$. Thus, increased placental calcium transfer in the Pthr1-null fetus was associated with a marked increase in the plasma level of PTHrP; the decreased placental calcium transfer in the Pthrp-null was associated with a reduction in plasma PTHrP; and the aparathyroid (PTH-less) Hoxa3-null had normal placental calcium transfer and normal plasma PTHrP levels.

Tissue sources of PTHrP in the fetus. The striking increase in plasma PTHrP level of the Pthr1-null fetuses enabled a focused search in this model to determine which tissue(s) might be overexpressing PTHrP and possibly contributing to the increased plasma level. The fetal parathyroids were too small to be isolated, and thus a whole neck section was used to represent the parathyroids. By Northern analysis of total RNA, a consistent increase in PTHrP mRNA was detected in placenta and liver of the Pthr1-null fetuses (Figure 3), but not in neck (Figure 3) or in head, lung, and umbilical cord (data not shown).

Next, sections of liver and placenta were examined by in situ hybridization and immunohistochemistry to determine which cell types were producing PTHrP. As seen in Figure 4, PTHrP mRNA was diffusely expressed in both wild-type and Pthr1-null placenta, but the signal intensi- ty was consistently increased in the Pthr1-null placenta. That this increase in mRNA resulted in increased production of protein was confirmed by immunohistochemistry, as seen in Figure 5. The peripheral and basal aspects of the placenta, which contains the spongiotrophoblasts and giant trophoblasts, had more intense PTHrP immunoreactivity than the labyrinthine (syncytial) trophoblasts that make up the bulk of the murine placenta (Figure 5e). This difference was maintained in the Pthr1-null placenta, but with more intensive PTHrP immunoreactivity in all three trophoblast cell types (compared with wild-type) (Figure 5, compare h with e). PTHrP immunoreactivity was also present in the intraplacental yolk sac (IPYS) of the wild-type and Ptbr1null placenta, more intensely in the columnar cells on the visceral side of the IPYS, and most intensely in the Pthr1null (Figure 5, compare $i$ with $\mathrm{f}$ ). In contrast to the findings in the Ptbr1-null placenta, no difference in PTHrP expression (mRNA or protein) was noted between wildtype and Hoxa3-null placentas (data not shown).

Examination of fetal liver showed the normal disarray of proliferating hematopoietic cells and liver cells, with expression of PTHrP in both lineages. PTHrP immunoreactivity was markedly increased in hematopoietic and liver cells of the Pthr1-null liver sections (Figure 6, compare $h$ and $i$ with $e$ and $f$ ). Thus, both placenta and liver were demonstrated to have increased expression of PTHrP (mRNA and protein) in the Ptbr1-null fetus, which has increased circulating levels of PTHrP.
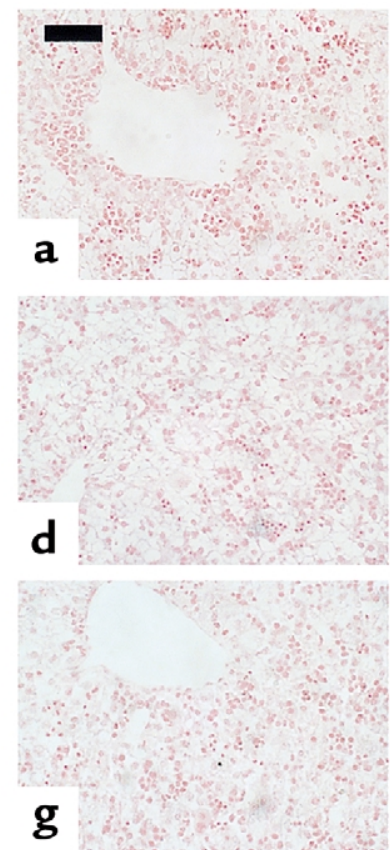
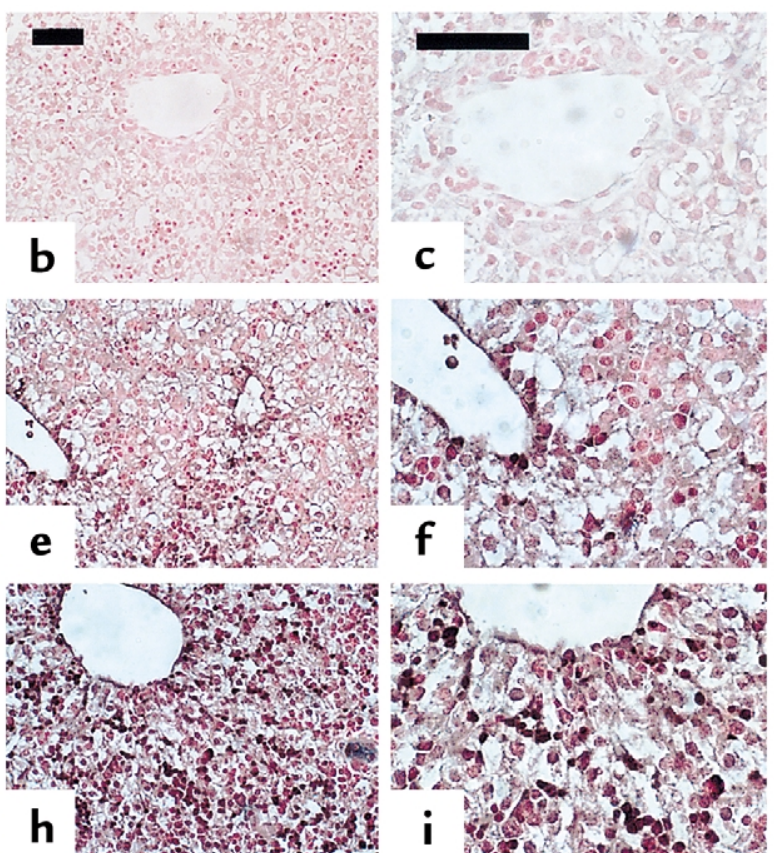

\section{Figure 6}

PTHrP immunohistochemistry of fetal liver. Upper images (a-c) are from Pthrp-null, middle images (d-f) are from wild-type, and lower images (g-i) are from Pthr1-null liver (ED 18.5). a, d, and $\mathbf{g}$ are control sections in which the primary antibody to PTHrP 1-34 was omitted. Pthrpnull sections are controls to demonstrate the antibody specificity. Normal extramedullary hematopoiesis and fetal liver cells are seen in all sections of fetal liver. Increased PTHrP immunoreactivity is demonstrated in liver and hematopoietic cells of Pthr1-null liver (h) compared with wild-type liver (e), especially in cells near central veins. Corresponding section of Pthrp-null (b) shows only nonspecific background. c, $\mathbf{f}$, and $\mathbf{i}$ are higher power images of the same sections in $\mathbf{b}, \mathbf{e}$, and $\mathbf{h}$, respectively. Calibration bars $=50 \mu \mathrm{m}$. 


\section{Discussion}

The present findings in aparathyroid Hoxa3-null fetuses demonstrate that these mice are functionally aparathyroid, as evidenced by absent PTH, reduced blood calcium, raised blood phosphate level, reduced serum magnesium, and reduced calcium in amniotic fluid. This confirms that the circulating level of PTH in fetal mice, though low, does have functional importance in regulating fetal calcium and magnesium metabolism. Also, the importance of Hoxa3 in parathyroid gland development and function is underscored by the finding that the circulating PTH level was reduced in $\mathrm{Hoxa}^{+/-}$fetuses. We have also found that the rate of placental calcium transfer and the plasma PTHrP level are unaffected in the Hoxa3-null fetuses. This result shows that the parathyroids are not required for the normal regulation of placental calcium transfer in mice, and that the parathyroids are not a major source of PTHrP in the circulation. Alternatively, it may be that other fetal tissue sources (liver, placenta) are compensating for the loss of PTHrP derived from the parathyroids in these null mice.

That these aparathyroid Hoxa3-null fetuses have normal placental calcium transfer contrasts with the previously reported finding of reduced placental calcium transfer in thyroparathyroidectomized fetal lambs. This may reflect a species difference between mice and sheep, including differences in structure and function of the placentas (hemochorial versus epitheliochorial, respectively). Alternatively, it could be due to experimental design. The present studies were carried out in intact fetal mice that lacked parathyroids from conception and whose mothers were heterozygous for the same genetic defect. The studies in fetal lambs involved a surgical procedure on the lambs, followed by later removal of the fetus and subsequent perfusion of the isolated placenta in situ $(4,20)$. Because plasma PTHrP measurements were not carried out for these thyroparathyroidectomized lambs, it is not known whether the reduction in placental calcium transfer was accompanied by a reduction in plasma PTHrP.

A limitation of the PTHrP RIA is that it detects a portion of the $\mathrm{NH}_{2}$-terminal region of PTHrP (PTHrP 134). The size of circulating PTHrP could not be determined, and yet it is likely that mid-molecular regions of the molecule stimulate placental calcium transfer in both mice and sheep. Given that the various fragments of PTHrP are thought to be derived from post-translational processing of full-length $\mathrm{PTHrP}$, an increase or decrease in PTHrP 1-34 concentration might be accompanied by a parallel change in the level of midmolecular and $\mathrm{COOH}$-terminal fragments of PTHrP. However, it is possible that the expression, metabolism and excretion of these fragments might be differentially regulated. Thus, a reduction in circulating midmolecular PTHrP cannot be completely excluded in the Hoxa3-null fetuses, as this was not specifically measured. The $\mathrm{NH}_{2}$-terminal assay was used because it is sensitive and has been well-characterized. By contrast, no well-characterized assays of mid-molecular PTHrP are available.

The in situ hybridization studies do not share this limitation since a cDNA for the entire PTHrP mRNA was used. No downregulation of PTHrP mRNA expression was noted in the Hoxa3-null fetuses, whereas upregulation of PTHrP mRNA was found in specific tissues in the Pthr1-null fetuses. Our findings indicate that PTHrP mRNA and protein expressed in placenta and liver are upregulated in response to loss of the $\mathrm{PTH} / \mathrm{PTHrP}$ receptor. These findings suggest that liver and placenta might be at least partly responsible for the increased circulating level of PTHrP in the Pthr1-null mice. It remains to be determined whether these tissues contribute to the circulating PTHrP level in normal circumstances.

In summary, our findings demonstrate that aparathyroid Hoxa3-null fetuses have absent PTH, reduced ionized calcium and serum magnesium, and raised serum phosphate, but normal plasma PTHrP and normal rate of placental calcium transfer. In contrast, Pthr1-null fetuses, which have an increased rate of placental calcium transfer, also have an 11-fold increase in circulating PTHrP level. Both liver and placenta (but not the neck or parathyroids) show increased expression of PTHrP mRNA and protein. The results indicate that the fetal parathyroids are required for maintenance of normal serum calcium, magnesium, and phosphate homeostasis, but are not required for maintenance of normal placental calcium transfer. Presumably, PTH acts on fetal bone and perhaps kidney to raise blood calcium. Furthermore, the parathyroids do not appear to contribute significantly to the circulating PTHrP level of fetuses. Instead, other fetal tissue sources, especially placenta and liver, may be more important for regulating fetal calcium homeostasis than previously suspected.

It is now apparent that both PTHrP and PTH are important for normal fetal calcium homeostasis, and that lack of either of these molecules will lead to disruptions in calcium metabolism (such as hypocalcemia and hyperphosphatemia), whereas only lack of PTHrP will reduce the rate of placental calcium transfer.

\section{Acknowledgments}

The authors thank Anthony D. Care for helpful discussions and suggestions. The technical assistance of Linda L. Chafe, Mandy L. Woodland, Kirsten R. McDonald, and Neva Fudge (Memorial University of Newfoundland), and Patricia Ho (University of Melbourne) is acknowledged. This work was supported by an NIH grant (DK-47038 to H.M. Kronenberg) and by a Fellowship award, a Scholarship award (MSH 35674), and an operating grant (MT-15439), all from the Medical Research Council of Canada to C.S. Kovacs. Additional research support (to C.S. Kovacs) was obtained from the Medical Research Foundation, the Research and Development Committee, the Faculty of Medicine, and the Discipline of Medicine, all at Memorial University of Newfoundland. 
1. Kovacs, C.S., and Kronenberg, H.M. 1997. Maternal-fetal calcium and bone metabolism during pregnancy, puerperium and lactation. Endocr Rev. 18:832-872.

2. Karaplis, A.C., et al. 1994. Lethal skeletal dysplasia from targeted disruption of the parathyroid hormone-related peptide gene. Genes. Dev. 8:277-289

3. Kovacs, C.S., et al. 1996. Parathyroid hormone-related peptide (PTHrP) regulates fetal-placental calcium transport through a receptor distinct from the PTH/PTHrP receptor. Proc. Natl. Acad. Sci. USA. 93:15233-15238.

4. Abbas, S.K., et al. 1989. Stimulation of ovine placental calcium transport by purified natural and recombinant parathyroid hormone-related protein (PTHrP) preparations. Q. J. Exp. Physiol. 74:549-552.

5. Care, A.D., et al. 1990. Stimulation of ovine placental transport of calcium and magnesium by mid-molecule fragments of human parathyroid hormone-related protein. Exp. Physiol. 75:605-608.

6. Lanske, B., et al. 1996. PTH/PTHrP receptor in early development and Indian hedgehog-regulated bone growth. Science. 273:663-666.

7. Kovacs, C.S., Lanske, B., Byrne, M., Krane, S.M., and Kronenberg, H.M. 1997. Altered interstitial collagenase expression in the tibias of PTHrP gene-ablated and PTH/PTHrP receptor gene-ablated fetal mice. J. Bone Miner. Res. 12(Suppl.):S116. (Abstr.)

8. Ho, C., et al. 1995. A mouse model of human familial hypocalciuric hypercalcemia and neonatal severe hyperparathyroidism. Nat. Genet. 11:389-394.

9. Kovacs, C.S., et al. 1998. Regulation of murine fetal-placental calcium metabolism by the calcium-sensing receptor. J. Clin. Invest. 101:2812-2820.

10. Tucci, J., et al. 1996. The expression of parathyroid hormone and parathyroid hormone-related protein in developing rat parathyroid glands. J. Mol. Endocrinol. 17:149-157.
11. Philbrick, W.M., et al. 1996. Defining the roles of parathyroid hormonerelated protein in normal physiology. Physiol. Rev. 76:127-173.

12. Manley, N.R., and Capecchi, M.R. 1995. The role of Hoxa-3 in mouse thymus and thyroid development. Development. 121:1989-2003.

13. Chisaka, O., and Capecchi, M.R. 1991. Regionally restricted developmental defects resulting from targeted disruption of the mouse homeobox gene hox-1.5. Nature. 350:473-479.

14. Lavigne, J.R., et al. 1997. Development and use of a new, highly-sensitive immunoradiometric assay (IRMA) specific for rat calcitonin. Proceedings of the 79th Annual Meeting of the Endocrine Society. June 11-14, 1997, Minneapolis, Minnesota, USA. Endocrine Society. Bethesda, Maryland, USA. p. 243. (Abstr.)

15. Grill, V., et al. 1991. Parathyroid hormone-related protein: elevated levels in both humoral hypercalcemia of malignancy and hypercalcemia complicating metastatic breast cancer. J. Clin. Endocrinol. Metab. 73:1309-1315.

16. Ausubel, F.M., et al. 1997. Placental perfusion of adult mice. In Current protocols in molecular biology. Volume 2. F.M. Ausubel et al., editors. John Wiley \& Sons. Boston, Massachusetts, USA. p. 14.1.4.

17. Lanske, B., et al. 1998. The parathyroid hormone/parathyroid hormonerelated peptide receptor mediates actions of both ligands in murine bone. Endocrinology. 139:5192-5204.

18. Kartsogiannis, V., et al. 1997. Temporal expression of PTHrP during endochondral bone formation in mouse and intramembranous bone formation in an in vivo rabbit model. Bone. 21:385-392.

19. Günther, T., et al. 2000. Genetic ablation of parathyroid glands reveals another source of parathyroid hormone. Nature. 406:199-203.

20. Care, A.D., Caple, I.W., Abbas, S.K., and Pickard, D.W. 1986. The effect of fetal thyroparathyroidectomy on the transport of calcium across the ovine placenta to the fetus. Placenta. 7:417-424. 